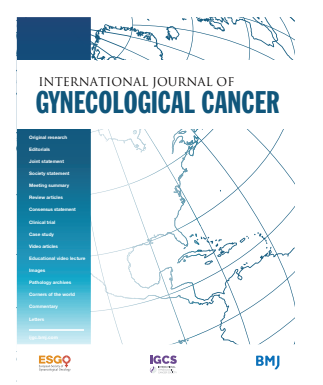

\title{
Efficacy of pre-operative pharmacologic thromboprophylaxis on incidence of venous thromboembolism following major gynecologic and gynecologic oncology surgery: a systematic review and meta- analysis
}

Steven Bisch, ${ }^{1}$ Rachelle Findley (D),${ }^{1}$ Christina Ince, ${ }^{1}$ Maria Nardell, ${ }^{2}$ Gregg Nelson ${ }^{1}$

- Additional material is published online only. To view please visit the journal online (http://dx.doi.org/10.1136/ijgc2020-001991).

For numbered affiliations see end of article.

\section{Correspondence to} Dr Rachelle Findley, Gynecologic Oncology, Tom Baker Cancer Centre, Calgary, AB T2N 4N2, Canada; rachelle. findley@albertahealthservices. ca

$\mathrm{SB}$ and $\mathrm{RF}$ are joint first authors.

Received 22 August 2020 Revised 28 0ctober 2020 Accepted 30 October 2020 Published Online First 19 November 2020

\section{Check for updates}

(C) IGCS and ESGO 2021. No commercial re-use. See rights and permissions. Published by BMJ.

To cite: Bisch S, Findley R, Ince C, et al. Int J Gynecol Cancer 2021;31:257-264.

\section{HIGHLIGHTS}

- The analysis included one randomized controlled trial and 11 cohort studies for a total of 14273 patients.

- Use of pre-operative chemoprophylaxis reduced venous thromboembolism with an OR of 0.59 .

- Reduction in venous thromboembolism was more pronounced when combined with mechanical prophylaxis.

\section{ABSTRACT}

Introduction Venous thromboembolism remains a significant complication following major gynecologic surgery. Evidence is lacking on whether it is beneficial to give pharmacologic thromboprophylaxis pre-operatively. The aim of this meta-analysis was to assess the role of pre-operative pharmacologic thromboprophylaxis in preventing post-operative venous thromboembolism. Methods PubMed, EMBASE, and the Cochrane Central Register of Clinical Trials were searched to find randomized controlled, cohort, and case-control trials comparing pre-operative pharmacologic thromboprophylaxis to no prophylaxis, mechanical prophylaxis, or only post-operative pharmacologic thromboprophylaxis for open and minimally invasive major gynecologic surgery (benign and malignant conditions). Two authors independently assessed abstracts, full-text articles, and methodological quality. Data were extracted and pooled using ORs for random effects metaanalysis. Heterogeneity was explored using forest plots, Qstatistic, and $\mathrm{I}^{2}$ statistics. Planned subgroup analysis of use of sequential compression devices, equivalent versus nonequivalent post-operative prophylaxis, cancer diagnosis, and methodological quality were performed.

Results Some 503 unique studies were found, and 16 studies (28806 patients) were included in the systematic review. Twelve studies (14273 patients) were included in the meta-analysis. The $\mathrm{OR}$ for incidence of post-operative venous thromboembolism was $0.59(95 \% \mathrm{Cl} 0.39,0.89)$, favoring pre-operative pharmacologic thromboembolism prophylaxis compared with no pre-operative pharmacologic prophylaxis $\left(Q=13.80, I^{2}=20.30\right)$. In studies where post-operative care was equivalent between groups, the $\mathrm{OR}$ for venous thromboembolism was $0.56(95 \% \mathrm{Cl}$ $0.22,1.40)$. Pre-operative pharmacologic prophylaxis demonstrated greatest benefit when utilized with both intra-operative and post-operative sequential compression devices (OR $0.43,95 \% \mathrm{Cl} 0.30,0.64)$ compared with when no sequential compression devices were utilized (OR $1.27,95 \% \mathrm{Cl} 0.63,2.56)$. When looking at only studies determined to be of high quality, the results no longer reached significance $(0 \mathrm{R} 0.73,95 \% \mathrm{Cl} 0.36,1.46)$.

Conclusions Pre-operative pharmacologic thromboprophylaxis decreases the odds of venous thromboembolism in the peri-operative period for major gynecologic oncology surgery by approximately $40 \%$. It remains unclear whether this benefit is present in benign and minor procedures. Adequately powered studies are needed.

\section{INTRODUCTION}

Deep vein thrombosis and pulmonary embolism (collectively referred to as venous thromboembolism) are common complications following major gynecologic surgery. The rate of deep vein thrombosis after gynecologic surgery without prophylaxis is approximately $16 \%-38 \%$, while the rate of pulmonary embolism is close to $1 \% .^{12}$ Surgery is a wellrecognized risk factor for venous thromboembolism. Additional risk factors include advancing age, malignancy, obesity, acute medical illness, and prior venous thromboembolism. Unfortunately, exact definitions of major versus minor surgical procedures are lacking within the literature. ${ }^{3}$ Guidelines are available from a variety of associations providing practice recommendations for peri-operative thromboprophylaxis (American College of Chest Physicians; American College of Obstetricians and Gynecologists, ENDORSE study). ${ }^{3-7}$ Despite numerous guidelines, the international study ENDORSE, which analyzed the administration of appropriate thromboprophylaxis in patients admitted for medical and surgical indications, found 


\section{Original research}

that among patients at risk for venous thromboembolism only $39.5 \%$ of medical and $58.5 \%$ of surgical inpatients received appropriate prophylaxis. ${ }^{4}$ Similarly, another USA registry looked at 5451 patients with ultrasound-confirmed deep vein thrombosis from 183 different sites across the country. This study found that only 1142 $(42 \%)$ patients received the indicated prophylaxis. ${ }^{5}$ In the same study, $32 \%$ of patients diagnosed with a deep vein thrombosis had surgery during the prior 3 months. It is estimated that thromboprophylaxis for at-risk inpatients can reduce venous thromboembolism by $30 \%$ to $65 \%$ with a low incidence of major bleeding complications and well-documented cost-effectiveness. ${ }^{3}$

In an effort to encourage hospital-based quality improvement initiatives and protocols, the Agency for Healthcare Research and Quality provided recommendations on prevention of hospitalacquired venous thromboembolism. This guideline identified thromboprophylaxis as the "number one patient safety practice". 8 However, there are still many unanswered questions about pharmacologic thromboprophylaxis for venous thromboembolism. Guidelines from the American College of Chest Physicians state that most recommendations are based on low-quality evidence and call for further research in this area. ${ }^{6}$ One unresolved issue is the optimal timing for administration of the initial heparin dose in perioperative pharmacologic thromboprophylaxis. ${ }^{7}$ It's reported that nearly $50 \%$ of venous thromboemboli occur in the first 24 hours post-operatively and $75 \%$ begin within 72 hours of surgery. ${ }^{19}$ The American College of Obstetricians and Gynecologists Practice Bulletin 84 discusses that the optimal timing for initiation of perioperative pharmacologic thromboprophylaxis is unresolved. The necessity to initiate both mechanical and pharmacologic prophylaxis pre-operatively is weighed against the surgeon's concern of increased risk for intra-operative bleeding. ${ }^{7}$ The American College of Chest Physicians states that in the overwhelming majority of trials that demonstrated efficacy, unfractionated heparin and low molecular weight heparin were given 2 hours pre-operatively. While low molecular weight heparin also appears to be effective and is possibly associated with a lower risk of bleeding when the first dose is given 12 hours pre-operatively, specific recommendations on when to initiate prophylaxis are lacking. ${ }^{6}$

The aim of our study was to assess existing evidence on the efficacy of pharmacologic thromboprophylaxis initiated pre-operatively for major gynecologic and gynecologic oncology surgery in reducing venous thromboembolism to guide future clinical practice and improve patient safety and outcomes. The secondary objective was to confirm if the effectiveness of pre-operative pharmacologic thromboprophylaxis was impacted by surgical indication (benign or malignant), type of medication used, and the post-operative use of pharmacologic or mechanical thromboprophylaxis.

\section{METHODS}

This review followed Preferred Reporting Items for Systematic Reviews and Meta-Analyses (PRISMA) guidelines for systematic review and meta-analyses.

\section{Study Eligibility Criteria}

Eligible studies included English-language randomized controlled trials, cohort studies, and case-control studies that compared pre-operative pharmacologic thromboprophylaxis with or without mechanical prophylaxis to either no prophylaxis, mechanical prophylaxis, or only post-operative pharmacologic thromboprophylaxis. A required outcome was newly diagnosed post-operative venous thromboembolism within 1 month of surgery. Eligible studies included open and minimally invasive major gynecologic surgery for benign and malignant conditions with hospital admission. Studies were excluded if venous thromboembolism was not diagnosed by ultrasound doppler (deep vein thrombosis) or computed tomography or ventilation perfusion scan (pulmonary embolism) or if the data were in a form that was not usable for meta-analysis. Studies involving obstetric surgery (such as Cesarean section) and minor procedures not requiring hospital admission were excluded. Data from peer-reviewed abstracts were included if the necessary values were presented given the relative paucity of data available on the topic. ${ }^{10}$ Sensitivity analysis without included abstracts was also performed. Study protocols, letters to the editor, non-peer reviewed publications, case series, case reports, and non-controlled studies were excluded from analysis.

\section{Identification and Selection of Studies}

PubMed, EMBASE, and The Cochrane Central Register of Clinical Trials were searched for all studies meeting eligibility criteria. The search strategy utilized Medical Subject Headings (MeSH) terms "thromboembolism", "anticoagulants", "heparin", "gynecology", "genital neoplasms, female/surgery", "obstetrics" in addition to Boolean search strategies (available in online supplemental appendix 1). Two reviewers independently assessed abstracts and full-text articles. Disagreements were resolved through consensus or by a third reviewer.

\section{Data Collection and Study Appraisal}

Two independent reviewers extracted data on the type of preoperative pharmacologic thromboprophylaxis (unfractionated heparin or low molecular weight heparin), duration of prophylaxis, post-operative use, use of sequential compression devices, and whether the participants had benign or malignant disease. Outcome data included number of venous thromboembolism events in the intervention group and the control group and/or the OR for venous thromboembolism events.

Quality of each observational study was determined using the Newcastle-0ttawa Scale and the Agency for Healthcare Research and Quality Tool. "Good quality" was defined as 3-4 stars in the selection domain, 1-2 stars in the comparability domain, and 2-3 stars in the outcome/exposure domain. "Fair quality" was assigned if a study had 2 stars for selection, 1-2 stars for comparability, and 2-3 stars for outcome/exposures. "Poor quality" studies had 0-1 stars in selection, 0 stars in comparability, or 0-1 stars in outcome/ exposure. Randomized trials were reviewed by Jadad score. ${ }^{11}$ Two reviewers independently assessed bias assessments, with disagreements resolved through consensus or by a third reviewer.

\section{Synthesis}

Data were pooled using ORs for random effects meta-analysis. Heterogeneity was explored using forest plots, Q-statistic, and $\mathrm{I}^{2}$ statistics. Clinical heterogeneity was assessed by a priori designated subgroup analysis. Subgroup analysis was undertaken by type of heparin, combination of pre-operative and post-operative use of pharmacologic thromboprophylaxis versus post-operative 
pharmacologic thromboprophylaxis alone, benign or malignant condition, use of both intra-operative and post-operative (until discharge or ambulating well) sequential compression devices, and quality. Where data were not available to pool in groups (eg, type of heparin not specified), they were not included in that subgroup analysis and those studies were described qualitatively. Statistical analyses were performed using Comprehensive Meta-Analysis (Biostat, Englewood, New Jersey, USA). Secondary analysis of bleeding complications (peri-operative blood transfusion, intraoperative blood loss greater than 1000 milliliters, post-operative vaginal vault bleed, post-operative pelvic hematoma) was also performed using random effects meta-analysis. In accordance with the Journal's guidelines, we will provide our data for the reproducibility of this study in other centers if such is requested.

\section{RESULTS}

We found 427 studies on PubMed and 131 on EMBASE based on our search criteria. Review of the Cochrane Central Register of Clinical trials did not yield any additional studies. Reasons for exclusion and the complete process of study selection are shown in Figure 1. Sixteen studies met our selection criteria and were initially included in our review. The 16 studies were evaluated for quality according to the Newcastle-0ttawa Scale and we decided to exclude the study by Swenson et al. ${ }^{12}$ This study utilized a stepwise regression algorithm for hypothesis generation about risk factors for venous thromboembolism. Given the nature of stepwise selection algorithms, the corrected $\mathrm{OR}$ for pre-operative pharmacologic thromboprophylaxis was not significant in the model, and the raw data were unavailable for re-analysis. The study by Piver et al was not included in the meta-analysis because no events were reported either in the intervention or in the control group. ${ }^{13}$ The study by Renata et al was excluded from the meta-analysis as the data from the abstract were unusable for meta-analysis $(95 \% \mathrm{Cl}$ was asymmetric). ${ }^{14}$ Data from Moore et al are reflected in the study by Whitworth et al and therefore were removed as a duplicate. ${ }^{15}$ Sensitivity analysis including the data from Swenson et al, with exclusion of the abstract-only data, ${ }^{16}{ }^{17}$ is also presented.

Meta-analysis of pooled data included 12 studies, which enrolled a total of 14273 patients. ${ }^{216-26}$ All but one of the analyzed studies included patients with gynecologic cancer; seven only included cancer patients and the four remaining studies included patients with malignant or benign diseases. There was only one randomized controlled trial by Maxwell et al. ${ }^{2}$ In this study, 228 patients were randomized to receive pre-operative low molecular weight heparin or to receive external pneumatic compression, and the main endpoint was the occurrence of venous thromboembolism. Complete data were available for 211 patients enrolled on this study. The main weakness of this study was lack of blinding. All the remaining studies were cohorts, eight retrospective ${ }^{16-2225}$ and three prospective. ${ }^{2324}{ }^{26}$ Characteristics of the 12 included studies are presented in Table 1.

Visual inspection of the funnel plot (Figure 2) shows that all included studies came within the expected confidence intervals, without suggestion of publication bias (Egger's regression intercept $=0.78,95 \% \mathrm{Cl}-0.87,2.43, \mathrm{p}=0.32$ ). According to the Newcastle-0ttawa Scale, in conjunction with the Agency for
Healthcare Research and Quality tool, five studies were classified as poor quality, ${ }^{16171^{19-21}}$ one study as fair quality, ${ }^{2}$ and six studies as high quality ${ }^{1822-26}$ (Table 2 - online supplemental material).

In our random effects pooled meta-analysis we found 67 events in the intervention groups $(n=6736)$ and 125 events in the control groups ( $n=7537)$, with an $\mathrm{OR}$ of 0.59 and a $95 \% \mathrm{Cl}$ from 0.39 to 0.89 (Figure 3), favoring the use of pre-operative pharmacologic venous thromboembolism prophylaxis. Minimal heterogeneity was found between studies $(Q=13.8$ with 11 degrees of freedom, $I^{2}=20.30$ ). Sensitivity analysis including the study by Swenson et al and without abstract-only data ${ }^{16}{ }^{17}$ demonstrates an OR of 0.80 with a $95 \% \mathrm{Cl}$ from 0.43 to 1.52 for the risk of thromboembolism with the use of pre-operative pharmacologic thromboprophylaxis compared with no pre-operative thromboprophylaxis.

The first subgroup analysis compared studies that only included cancer patients and studies with patients with both malignant and benign diseases. We found an $\mathrm{OR}$ of $0.73(95 \% \mathrm{Cl} 0.42,1.26)$ for the studies with only cancer patients and an $\mathrm{OR}$ of $0.36(95 \% \mathrm{Cl} 0.21$, 0.62 ) in the studies with a mixed population (Figure 4). Our second subgroup analysis compared the pre-operative use of unfractionated heparin and low molecular weight heparin. In this setting, we found an $\mathrm{OR}$ of $0.54(95 \% \mathrm{Cl} 0.31,0.94)$ in the unfractionated heparin group and an $\mathrm{OR}$ of 0.56 in the low molecular weight heparin group $(95 \% \mathrm{Cl}$ $0.07,4.49$ ) (online supplemental figure 5). The third subgroup analysis evaluated the role of pre-operative pharmacologic venous thromboembolism controlling for equivalent post-operative prophylaxis or with unequal post-operative venous thromboembolism prophylaxis. The result of this pooled analysis demonstrated an $\mathrm{OR}$ of $0.56(95 \% \mathrm{Cl}$ $0.22,1.40$ ) when equivalent post-operative venous thromboembolism prophylaxis is used between groups. The $\mathrm{OR}$ when post-operative venous thromboembolism prophylaxis is not equivalent between groups is $0.61(95 \% \mathrm{Cl} 0.37,1.02)$ (online supplemental figure 6). The fourth subgroup analysis divided the studies based on the use of sequential compression devices. In this analysis, the group of studies that employed sequential compression devices resulted in an $\mathrm{OR}$ of

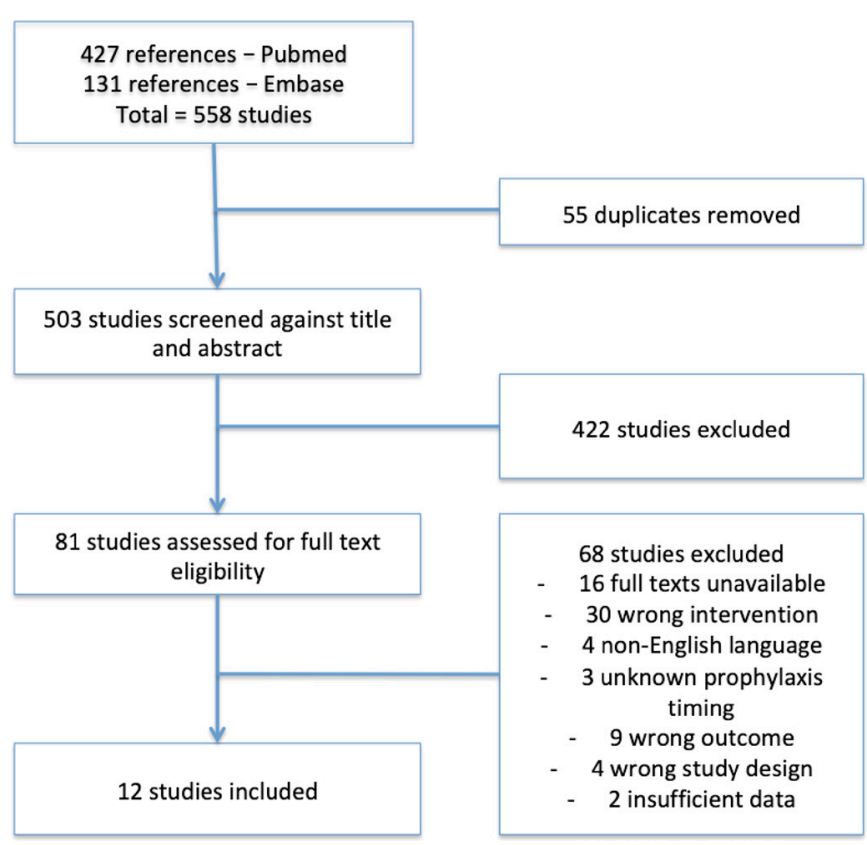

Figure 1 Study flow diagram. 


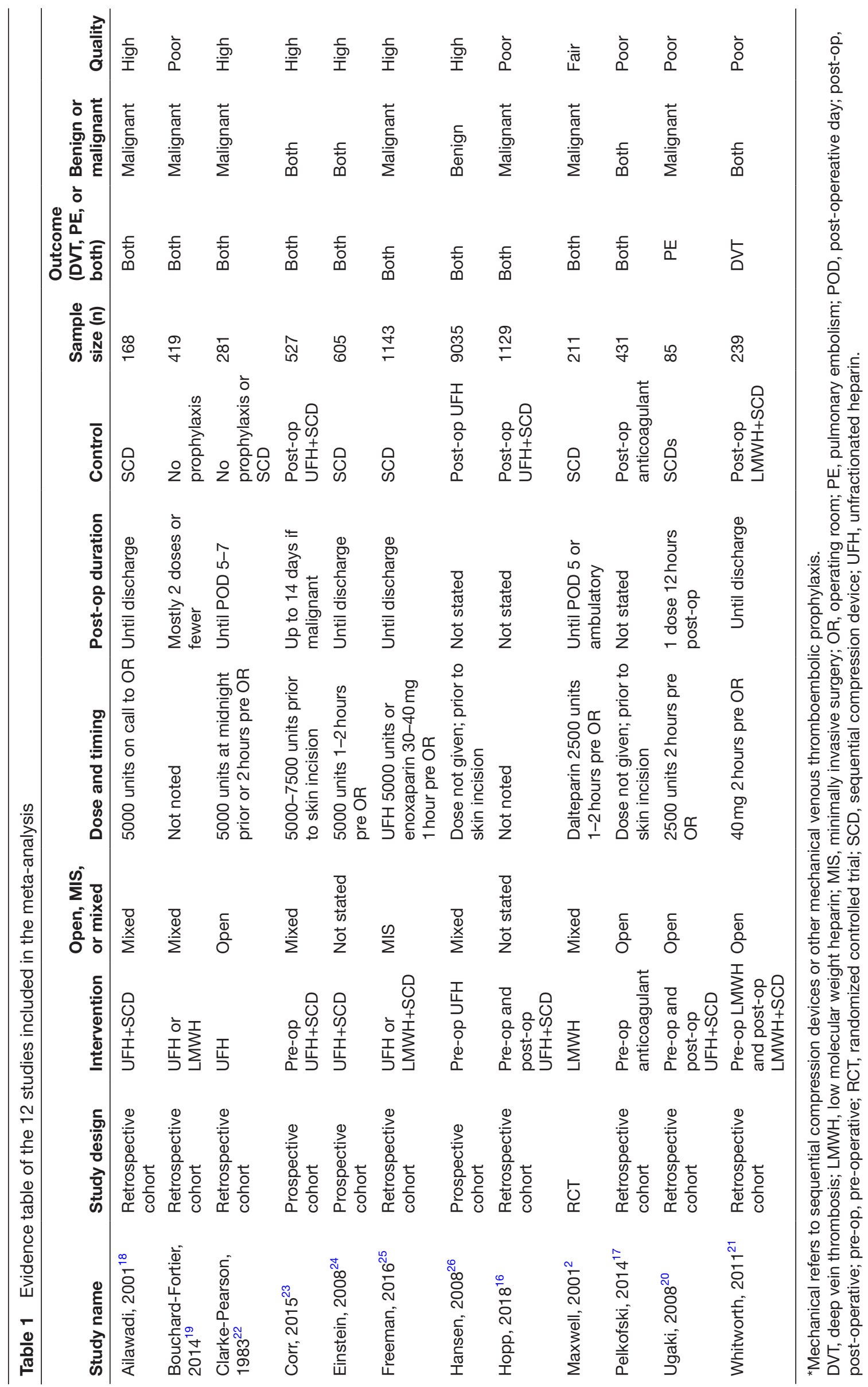

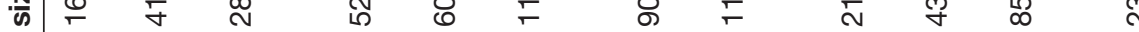

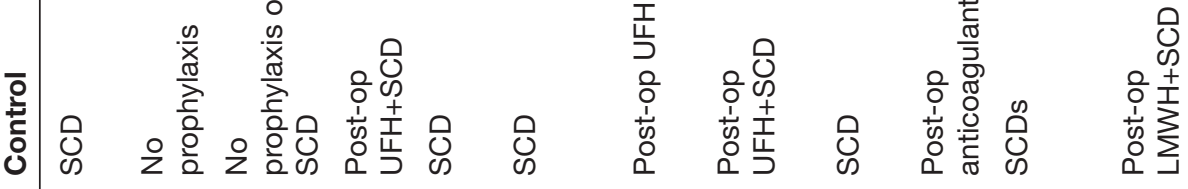

㟧宽

岕

N

웅

9

ठํ

궁

N 
Funnel Plot of Standard Error by Log odds ratio

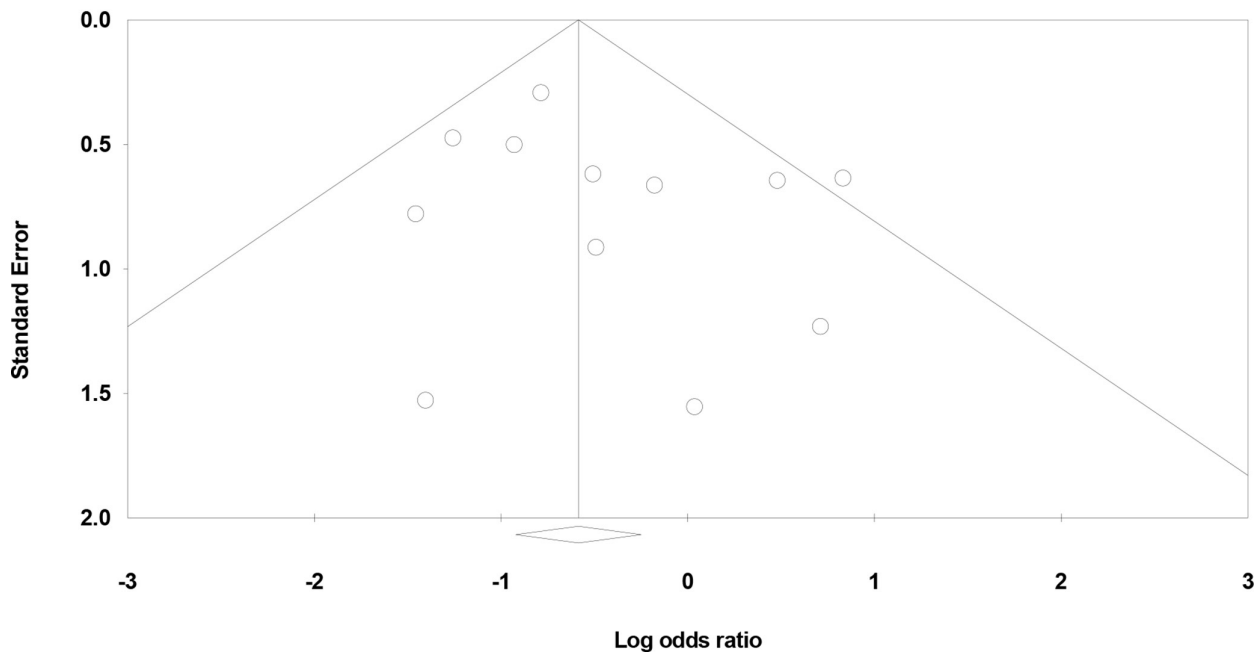

Figure 2 Funnel plot.

$0.43(95 \% \mathrm{Cl} 0.30,0.64)$ favoring the combination of pre-operative pharmacologic thromboprophylaxis in addition to sequential compression devices to sequential compression devices alone. The group of studies that did not use sequential compression devices resulted in an OR of $1.27(95 \% \mathrm{Cl} 0.63,2.56)$ in favor of pre-operative pharmacologic thromboprophylaxis (online supplemental figure 7). Our final subgroup analysis grouped patients according to their quality evaluations. When pooling the analyses for the high-quality studies only the OR was $0.73(95 \% \mathrm{Cl} 0.36,1.46)$ while the $\mathrm{OR}$ for poor-quality studies was $0.45(95 \% \mathrm{Cl} 0.28,0.72)$ (online supplemental figure 8$)$.

Data on the risk of bleeding complications associated with perioperative pharmacologic thromboprophylaxis was presented in nine papers (16409 patients). Pre-operative pharmacologic thromboprophylaxis was not associated with a significantly increased risk of perioperative bleeding complications with an OR of $1.26(95 \% \mathrm{Cl} 0.98$, 1.62).

\section{DISCUSSION}

\section{Summary of Main Results}

In this meta-analysis we demonstrate that the use of pre-operative pharmacologic thromboprophylaxis in addition to post-operative pharmacologic thromboprophylaxis decreases the odds of post-operative

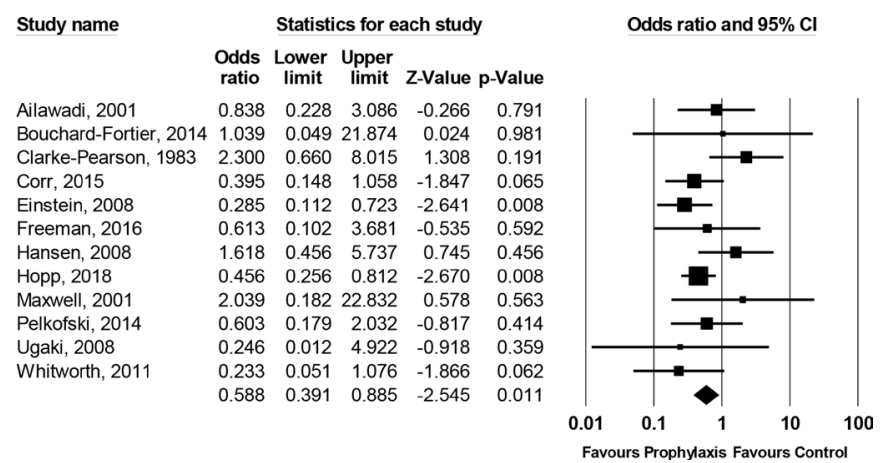

Figure 3 Meta-analysis of the odds of venous thromboembolism in studies of pre-operative pharmacologic thromboprophylaxis versus no pre-operative pharmacologic thromboprophylaxis. venous thromboembolism by just over $40 \%$. Our results are stable between both fixed and random effects models. Statistically there was minimal heterogeneity between studies, despite a priori concerns given the relative variability in dosage, type of prophylaxis, and surgery. Our analysis of publication bias demonstrated no obvious source of publication bias on the topic. Secondary analysis of bleeding complications trended towards an increased risk of bleeding complications with pre-operative pharmacologic thromboprophylaxis, although this result did not reach statistical significance $(p=0.07)$.

\section{Heterogeneity of Treatment Effect}

There was limited heterogeneity evident in our meta-analysis. The $Q$-statistic, $I^{2}$ value, and relative comparability of our fixed and random effects results suggest limited heterogeneity in the data. This can potentially be attributable to the homogeneity of the populations involved: women with malignancy who tend to present around a similar age (mid-60s). There was evidence of increased heterogeneity in the subgroup analysis of unfractionated and low molecular weight heparin. Although the mechanism of action of these drugs is similar, there may be differences in dosing and frequency that may contribute to variation in the results of studies. There is insufficient evidence to say that low molecular weight heparin produces similar protective effects as was seen for unfractionated heparin.

\section{Overall Completeness and Applicability of the Evidence}

A notable study that was excluded from this analysis is that of Swenson et al. ${ }^{12}$ This retrospective study of 20496 surgical patients demonstrated an increased unadjusted risk for venous thromboembolism with the use of pre-operative pharmacologic thromboprophylaxis. When the authors performed an adjusted analysis with potential confounders (malignancy, abdominal hysterectomy, duration of operation) this value was no longer statistically significant. The remaining $\mathrm{OR}$ for pre-operative pharmacologic thromboprophylaxis after this adjustment was unavailable for inclusion in the study due to the exploratory nature of the regression analysis used. On contacting the original authors, the raw data were unavailable for analysis. We hypothesize that since the result was no longer significant after adjustment for potential confounders, this study does 


\section{Original research}

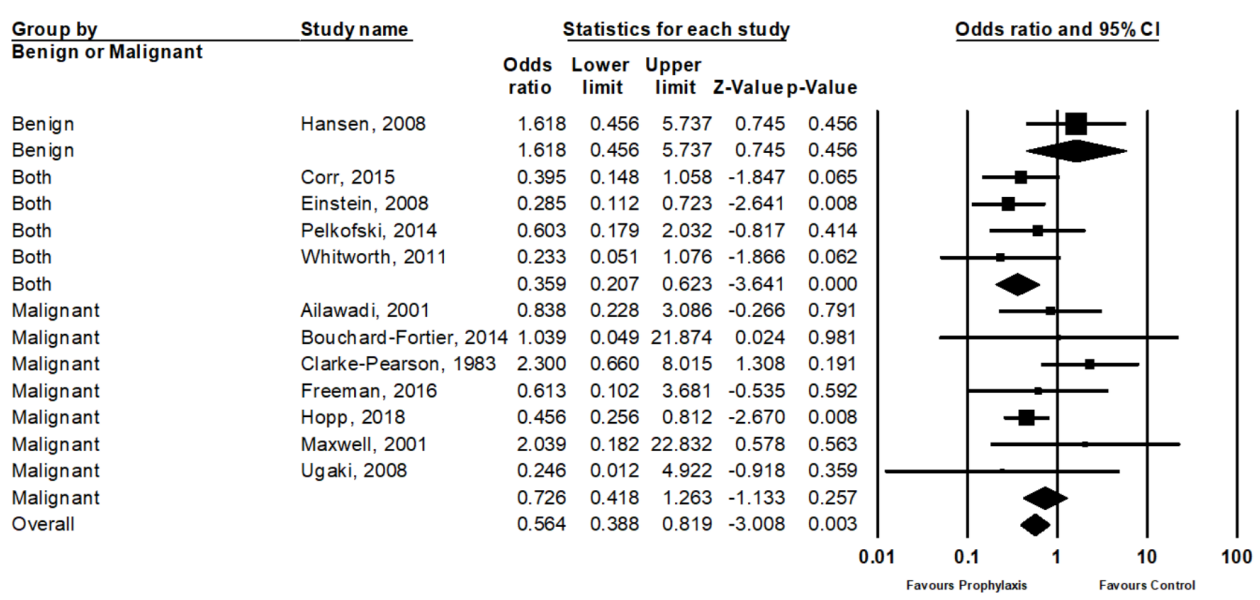

Figure 4 Forest plot of subgroup analysis comparing studies with cancer patients versus studies with mixed populations.

not refute our results. Unfortunately, a number of studies on the topic were done in the early 1970s and their full texts are not available for assessment. Additionally, there were a small number of trials (four) not published in English that were excluded from our study. English-language abstracts were available for three of these studies and, on secondary review, these studies would have been excluded due to inappropriate trial design or outcome measures.

\section{Quality of the Evidence}

Of the 12 trials included in this meta-analysis, only one (Maxwell et al) was a randomized controlled trial. ${ }^{2}$ The studies varied in their quality: six were of high quality with little risk of bias overall, one was fair quality, while five of the trials demonstrated major methodological flaws that risk the introduction of bias into their results. The greatest subgroup heterogeneity was demonstrated among the high-quality studies $\left(Q=10.07, I^{2}=50.3 \%\right)$, which included the manuscript by Hansen et al that focused on benign gynecology only. ${ }^{26}$

\section{Potential Bias in the Review Process}

The exclusion of older studies did not represent a majority of research on the topic as most studies were published recently. There was no evidence of publication bias on funnel plot on visual inspection or statistical testing (Egger's regression intercept $=0.78$, $95 \% \mathrm{Cl}-0.87,2.43, \mathrm{p}=0.32$ )

\section{Agreements and Disagreements with Other Studies or Reviews}

The study by Hansen et al presented the only study that focused on benign disease and demonstrated no significant effect of pre-operative pharmacologic thromboprophylaxis. ${ }^{26}$ Given the decreased risk of venous thromboembolism in patients with benign gynecologic disease compared with those with malignancy, this may represent a lack of power rather than a lack of effect. A recent Cochrane Review assessed the use of combined mechanical and pharmacologic prophylaxis at all times and for all surgeries. ${ }^{27} \mathrm{~A}$ subgroup analysis of combined pharmacologic and mechanical prophylaxis versus mechanical prophylaxis alone demonstrated an $O R$ of roughly 0.5 in favor of combined prophylaxis but the confidence intervals were large for prevention of pulmonary embolism. For the prevention of deep vein thrombosis, they did find an advantage to added pharmacologic prophylaxis. These differences can be explained by the inclusion of benign disease, as well as the inclusion of all patients and surgeries. The results support our findings of deep vein thrombosis prevention with combined pre-operative prophylaxis. A systematic review by Rahn et al in 2011 supports our findings of the benefit of combined pharmacologic and mechanical prophylaxis for gynecologic surgery at high-risk of venous thromboembolism. ${ }^{28}$ They also state that the evidence is insufficient to make strong recommendations for benign low-risk gynecologic surgery and that mechanical prophylaxis may be sufficient. ${ }^{28}$ The meta-analysis of prospective trials by Kakkos et al demonstrated a five-fold increased risk of bleeding with peri-operative pharmacologic prophylaxis. ${ }^{27}$ Our study looked only at pre-operative dose of pharmacologic thromboprophylaxis in the gynecologic population while Kakkos et al looked at pre- and post-operative thromboprophylaxis in urology, orthopedics, general surgery, as well as gynecologic surgery. The differences in population and intervention may account for the demonstrated increase in bleeding complications compared with our results.

\section{Implications for Practice and Research}

The majority of included studies involved surgery for gynecologic malignancy or a mixed population of benign and malignant indications. Only one study was included that involved surgery for only benign indications and this will limit the applicability of our findings in the benign population. Our findings suggest that the use of pharmacologic thromboprophylaxis prior to surgery is beneficial in patients undergoing surgery for gynecologic malignancy with a trend towards a small increased risk of surgical bleeding complications. Surgery for gynecologic malignancy carries considerable risk for thromboembolism as malignancy-related thrombophilia, venous compression by tumors, and prolonged and invasive surgery all contribute to a $20 \%-40 \%$ risk of post-operative thromboembolism in this population. The addition of low-dose pharmacologic thromboprophylaxis prior to surgical insult demonstrates a $41 \%$ reduction in the odds of this potentially life-threatening complication. The 2019 update of the Enhanced Recovery After Surgery (ERAS) Society guidelines for peri-operative care in gynecologic oncology reflects this finding by recommending that venous thromboembolism prophylaxis be initiated pre-operatively and continue in the post-operative period. ${ }^{29}$ 
This benefit of pre-operative pharmacologic thromboprophylaxis was most pronounced (on subgroup analysis) when used in combination with mechanical prophylaxis (intra-operative and postoperative sequential compression devices) with a $57 \%$ reduction in the odds of venous thromboembolism.

The role of pharmacologic thromboprophylaxis in benign gynecologic surgery is less clear and large, well-designed studies are necessary to determine the benefit of this practice in this population especially given the availability of mechanical prophylaxis. Further studies on the risk of peri-operative bleeding complications associated with pharmacologic thromboprophylaxis in gynecologic surgery are needed.

\section{CONCLUSIONS}

Pre-operative pharmacologic thromboprophylaxis decreases the risk of peri-operative venous thromboembolic complications. The decision to use pre-operative pharmacologic thromboprophylaxis should be based on the individual patient risk of thromboembolic events and their anticipated risk of major bleeding at time of surgery. Additional well-designed studies addressing this question are warranted.

\section{Author affiliations}

${ }^{1}$ Gynecologic Oncology, Tom Baker Cancer Centre, Calgary, Alberta, Canada ${ }^{2}$ Division of Global Health Equity, Brigham and Women's Hospital, Boston, Massachusetts, USA

Acknowledgements The authors would like to thank Drs Jovana Tavcar, Rodrigo Goncalves, and Mike Stoto for their contributions.

Contributors All authors reviewed the article for its content and editing. SB and MN formulated the idea for the systematic review and meta-analysis and contributed to the literature review, data collection, preparation, and editing of the article. $\mathrm{RF}$ and $\mathrm{Cl}$ contributed to the literature review, data collection, preparation, and editing of the article. GN contributed to the review and editing of the article.

Funding This publication was made possible by Grant Number T32 Al007433 from the National Institute of Allergy and Infectious Diseases and its contents are solely the responsibility of the authors and do not necessarily represent the official views of the National Institutes of Health.

Competing interests None declared.

Patient consent for publication Not required.

Provenance and peer review Not commissioned; externally peer reviewed.

Data availability statement All data relevant to the study are included in the article or uploaded as supplementary information.

Supplemental material This content has been supplied by the author(s). It has not been vetted by BMJ Publishing Group Limited (BMJ) and may not have been peer-reviewed. Any opinions or recommendations discussed are solely those of the author(s) and are not endorsed by BMJ. BMJ disclaims all liability and responsibility arising from any reliance placed on the content. Where the content includes any translated material, BMJ does not warrant the accuracy and reliability of the translations (including but not limited to local regulations, clinical guidelines, terminology, drug names and drug dosages), and is not responsible for any error and/or omissions arising from translation and adaptation or otherwise.

\section{ORCID iD}

Rachelle Findley http://orcid.org/0000-0002-6104-2779

\section{REFERENCES}

1 Clarke-Pearson DL, Synan IS, Colemen RE, et al. The natural history of postoperative venous thromboemboli in gynecologic oncology: a prospective study of 382 patients. Am J Obstet Gynecol 1984;148:1051-4.
2 Maxwell GL, Synan I, Dodge R, et al. Pneumatic compression versus low molecular weight heparin in gynecologic oncology surgery: a randomized trial. Obstet Gynecol 2001;98:989-95.

3 Geerts WH, Bergqvist D, Pineo GF, et al. Prevention of venous thromboembolism: American College of Chest Physicians evidence-based clinical practice guidelines (8th edition). Chest 2008;133:381S-453.

4 Cohen AT, Tapson VF, Bergmann J-F, et al. Venous thromboembolism risk and prophylaxis in the acute hospital care setting (ENDORSE study): a multinational cross-sectional study. Lancet 2008;371:387-94.

5 Goldhaber SZ, Tapson VF, DVT FREE Steering Committee. A prospective registry of 5,451 patients with ultrasound-confirmed deep vein thrombosis. Am J Cardiol 2004;93:259-62.

6 Gould MK, Garcia DA, Wren SM, et al. Prevention of VTE in nonorthopedic surgical patients: antithrombotic therapy and prevention of thrombosis (9th edition): American College of Chest Physicians evidence-based clinical practice guidelines. Chest 2012;141:e227S-77.

7 Committee on Practice Bulletins--Gynecology, American College of Obstetricians and Gynecologists. ACOG Practice Bulletin No. 84: prevention of deep vein thrombosis and pulmonary embolism. Obstet Gynecol 2007;110:429-40.

8 Agency for Healthcare Research and Quality. Preventing hospitalassociated venous thromboembolism: a guide for effective quality improvement; 2016. https://www.ahrq.gov/sites/default/files/ publications/files/vteguide.pdf

9 Kakkar VV, Howe CT, Flanc C, et al. Natural history of postoperative deep-vein thrombosis. The Lancet 1969;294:230-3.

10 Scherer RW, Saldanha IJ. How should systematic reviewers handle conference abstracts? A view from the trenches. Syst Rev 2019;8:264

11 Jadad AR, Moore RA, Carroll D, et al. Assessing the quality of reports of randomized clinical trials: is blinding necessary? Control Clin Trials 1996;17:1-12.

12 Swenson CW, Berger MB, Kamdar NS, et al. Risk factors for venous thromboembolism after hysterectomy. Obstet Gynecol 2015;125:1139-44.

13 Piver MS, Malfetano JH, Lele SB, et al. Prophylactic anticoagulation as a possible cause of inguinal lymphocyst after radical vulvectomy and inguinal lymphadenectomy. Obstet Gynecol 1983;62:17-21.

14 Renata R, Schiff M, Goff B. The impact of a comprehensive perioperative venous thromboembolism prophylaxis plan on a gynecologic oncology service. Gynecol Oncol 2013;131:274-5.

15 Moore J, Whitworth J, Matthews KS, et al. Double prophylaxis for deep venous thrombosis in gynecologic oncology patients undergoing laparotomy: does preoperative anticoagulation matter? Gynecol Oncol 2009;112:S171-2.

16 Hopp E, Martin L, Kumar J, et al. Incidence of venous thromboembolism in the gynecologic cancer patient population at a single institution after implementation of pre-operative venous thromboembolism prophylaxis. J Clin Oncol 2018;36.

17 Pelkofski E, Courtney-Brooks MB, Tanner Kurtz CK, et al. Use of a perioperative anticoagulation protocol in gynecologic oncology patients receiving continuous epidural analgesia. Gynecol Oncol 2014;133:140.

18 Ailawadi M, Del Priore G. A comparison of thromboembolic prophylaxis in gynecologic oncology patients. Int $J$ Gynecol Cancer 2001;11:354-8. doi:10.1046/j.1525-1438.2001.01045.x

19 Bouchard-Fortier G, Geerts WH, Covens A, et al. Is venous thromboprophylaxis necessary in patients undergoing minimally invasive surgery for a gynecologic malignancy? Gynecol Oncol 2014;134:228-32.

20 Ugaki H, Enomoto T, Fujiwara K, et al. Safety and efficacy of lowerdose unfractionated heparin for prophylaxis of deep vein thrombosis and pulmonary embolism in an Asian population. Blood Coagul Fibrinolysis 2008;19:585-9.

21 Whitworth JM, Schneider KE, Frederick PJ, et al. Double prophylaxis for deep venous thrombosis in patients with gynecologic oncology who are undergoing laparotomy: does preoperative anticoagulation matter? Int J Gynecol Cancer 2011;21:1131-4. doi:10.1097/ IGC.0b013e31821dc9f0

22 Clarke-Pearson DL, Jelovsek FR, Creasman WT. Thromboembolism complicating surgery for cervical and uterine malignancy: incidence, risk factors, and prophylaxis. Obstet Gynecol 1983;61:87-94.

23 Corr BR, Winter AM, Sammel MD, et al. Effectiveness and safety of expanded perioperative thromboprophylaxis in complex gynecologic surgery. Gynecol Oncol 2015;138:501-6.

24 Einstein MH, Kushner DM, Connor JP, et al. A protocol of dual prophylaxis for venous thromboembolism prevention in gynecologic cancer patients. Obstet Gynecol 2008;112:1091-7. 


\section{Original research}

25 Freeman $\mathrm{AH}$, Barrie A, Lyon L, et al. Venous thromboembolism following minimally invasive surgery among women with endometrial cancer. Gynecol Oncol 2016;142:267-72.

26 Hansen CT, Kehlet H, Møller C, et al. Timing of heparin prophylaxis and bleeding complications in hysterectomy a nationwide prospective cohort study of 9,949 Danish women. Acta Obstet Gynecol Scand 2008;87:1039-47.

27 Kakkos SK, Caprini JA, Geroulakos G, et al. Combined intermittent pneumatic leg compression and pharmacological prophylaxis for prevention of venous thromboembolism. Cochrane Database Syst Rev 2016;9.

28 Rahn DD, Mamik MM, Sanses TV, et al. Venous thromboembolism prophylaxis in gynecologic surgery: a systematic review. Obstet Gynecol 2011;118:1111-25.

29 Nelson G, Bakkum-Gamez J, Kalogera E, et al. Guidelines for perioperative care in gynecologic/oncology: Enhanced Recovery After Surgery (ERAS) Society recommendations-2019 update. Int J Gynecol Cancer 2019;29:651-68. 